\title{
MONITORAMENTO QUALITATIVO DA ÁGUA DO RIBEIRÃO ABÓBORA DO MUNICÍPIO DE RIO VERDE GO, COMO INSTRUMENTO DE GESTÃO DE RECURSOS HÍDRICOS
}

\section{QUALITATIVE MONITORING OF RIBEIRÃO PUMPKIN WATER IN THE CITY OF RIO VERDE GO, AS AN INSTRUMENT FOR WATER RESOURCE MANAGEMENT}

\section{Jonathan Oliveira Arantes \\ Engenheiro Ambiental pela UNIRV Universidade de Rio Verde, mestrando em Engenharia aplicada e sustentabilidade pelo IF Goiano de Rio Verde. E-mail:}

jhom111@hotmail.com

\section{Gilmar Oliveira Santos} Professor Adjunto da Universidade de Rio Verde (UniRV) com aulas em cursos de Graduação, PósGraduação e Mestrado E-mail:

gilmar@unirv.edu.br

\section{RESUMO}

O monitoramento da água uma ferramenta essencial na garantia da qualidade dos recursos hídricos. O presente trabalho teve por objetivo o monitoramento da água do ribeirão Abóbora do município de Rio Verde GO, como instrumento de gestão de recursos hídricos. Foram realizadas cinco coletas de água, que ocorreram a montante e a jusante do ponto de descarte de efluente industrial existente no trecho em estudo. A média geral dos valores de OD observados correspondeu a $6 \mathrm{mg} / \mathrm{L} \mathrm{e}$ $7 \mathrm{mg} / \mathrm{L}$ para os anos de 2016 e 2017 respectivamente. Os meses de outubro/16 no ponto "jusante" e novembro/16 nos pontos "montante e jusante" apresentaram concentrações de OD abaixo do valor exigido ( $\geq 5,0 \mathrm{mg} / \mathrm{L})$ pela norma Conama 357/2005 para rios classe II. A concentração média de $\mathrm{NO}_{2}{ }^{-}$nas amostras de água do manancial analisado foram de $0,1 \mathrm{mg} / \mathrm{L} \mathrm{e} 0,04 \mathrm{mg} / \mathrm{L}$ para os anos de $2016 \mathrm{e}$ 2017. Não houve ultrapassagem do limite legal $(\leq 1,0)$ preconizado pela norma Conama 357/05. Os resultados médios de $\mathrm{NO}_{3}^{-}$para toda a campanha de monitoramento foi de $0,3 \mathrm{mg} / \mathrm{L}$ para ambos os anos 2016 e 2017. Segundo o método abordado, quanto a variação dos dados observados o experimento pode ser classificado como de "boa precisão".

Palavras-chave: Ferramenta. Limite legal. Manancial.

\begin{abstract}
Water monitoring is an essential tool in ensuring the quality of water resources. The objective of the present work was to monitor the water of the Abeirão stream in the municipality of Rio Verde GO, as an instrument for managing water resources. Five water collections were carried out, which took place upstream and downstream of the industrial effluent disposal point in the stretch under study. The general average of the observed OD values corresponded to $6 \mathrm{mg} / \mathrm{L}$ and $7 \mathrm{mg} / \mathrm{L}$ for the years 2016 and 2017 respectively. The months of October / 16 at the "downstream" point and November / 16 at the "upstream and downstream" points presented DO concentrations below the required value ( $\geq 5.0 \mathrm{mg} / \mathrm{L}$ ) by the Conama 357/2005 standard for class II rivers. The average concentration of NO2- in the water samples from the analyzed source was $0.1 \mathrm{mg} / \mathrm{L}$ and $0.04 \mathrm{mg} / \mathrm{L}$ for the years 2016 and 2017. There was no exceedance of the legal limit $(\leq 1.0)$ recommended by the Conama standard 357/05. The average results of NO3- for the whole monitoring campaign was $0.3 \mathrm{mg} / \mathrm{L}$ for both years 2016 and 2017. According to the approached method, regarding the variation of the observed data, the experiment can be classified as "good accuracy.
\end{abstract}

Keywords: Tool. Legal limit. Source. 


\section{INTRODUÇÃO}

A qualidade das águas dos mananciais é uma condição ambiental essencial para sobrevivência dos seres humanos e aquáticos, pois está ligada a qualidade necessária para a potabilidade humana e a sobrevivência dos animais que vivem nos ambientes aquáticos. Assim, o correto tratamento da água assegura o seu uso como matéria prima essencial a maioria dos processos industriais e fabricações.

Tendo em mente o desenvolvimento econômico do Brasil nas últimas décadas, seja nas áreas urbanas ou rurais, é possível identificar um planejamento inadequado das bacias hidrográficas, com uma pressão cada vez maior sobre os recursos naturais, de forma especial os recursos hídricos (VANZELA; HERNANDEZ; FRANCO 2010).

Dessa forma, o monitoramento da qualidade da água surgiu da necessidade de assegurar a viabilidade dos diversos usos, influenciando de forma direta a gestão dos recursos hídricos e a busca por soluções de conflitos pelo uso. Nesse contexto, o monitoramento da qualidade da água pode ser definido como o processo de amostragem, de determinação de parâmetros de interesse e de armazenamento de dados contemplando as características físicas, químicas e biológicas das águas naturais (MAIA; SILVA; LIBANIO; 2019).

A compreensão a respeito do grau de poluição dos recursos hídricos pode auxiliar na antecipação a muitas condições indesejadas como "a eutrofização, mortandade de peixes, a interferência no tratamento da água visando o consumo humano e ao comprometimento a qualidade necessária aos usos múltiplos" a qual ela é destinada. Sendo assim, a preservação dos recursos hídricos requer um monitoramento adequado através de técnicas que sejam capazes de identificar o real estado da qualidade da água, e para esse propósito, a determinação de parâmetros físicos e químicos tem sido utilizada (MARANHO et al., 2017).

Nesse sentido o presente trabalho teve como objetivo verificar a qualidade da água do Ribeirão Abóbora e avaliar se os valores dos parâmetros obtidos estão em conformidade com os padrões estabelecidos pela legislação ambiental.

\section{MATERIAL E MÉTODOS}

O presente estudo foi realizado no Ribeirão das Abóboras, município de Rio Verde, região sudoeste do estado de Goiás, em um ponto abaixo da contribuição do Córrego Morimbondo próximo a captação da SANEAGO (Figura 1). Esse ponto é delimitado pelas coordenadas geográficas $17^{\circ} 44^{\prime} 25.25^{\prime \prime S}$ e $50^{\circ} 59^{\prime} 44.44^{\prime \prime O}$ correspondente ao início do manancial e $17^{\circ} 54^{\prime} 43.16^{\prime \prime} \mathrm{S}$ e $50^{\circ} 55^{\prime} .0 .79$ ' O referente ao fim desse corpo hídrico e ocupa uma área total de $205 \mathrm{~km}^{2}$ (GARCIA et al., 2007). 
Figura 1. Localização dos pontos de estudo na microbacia hidrográfica do Ribeirão das Abóboras, 2017.

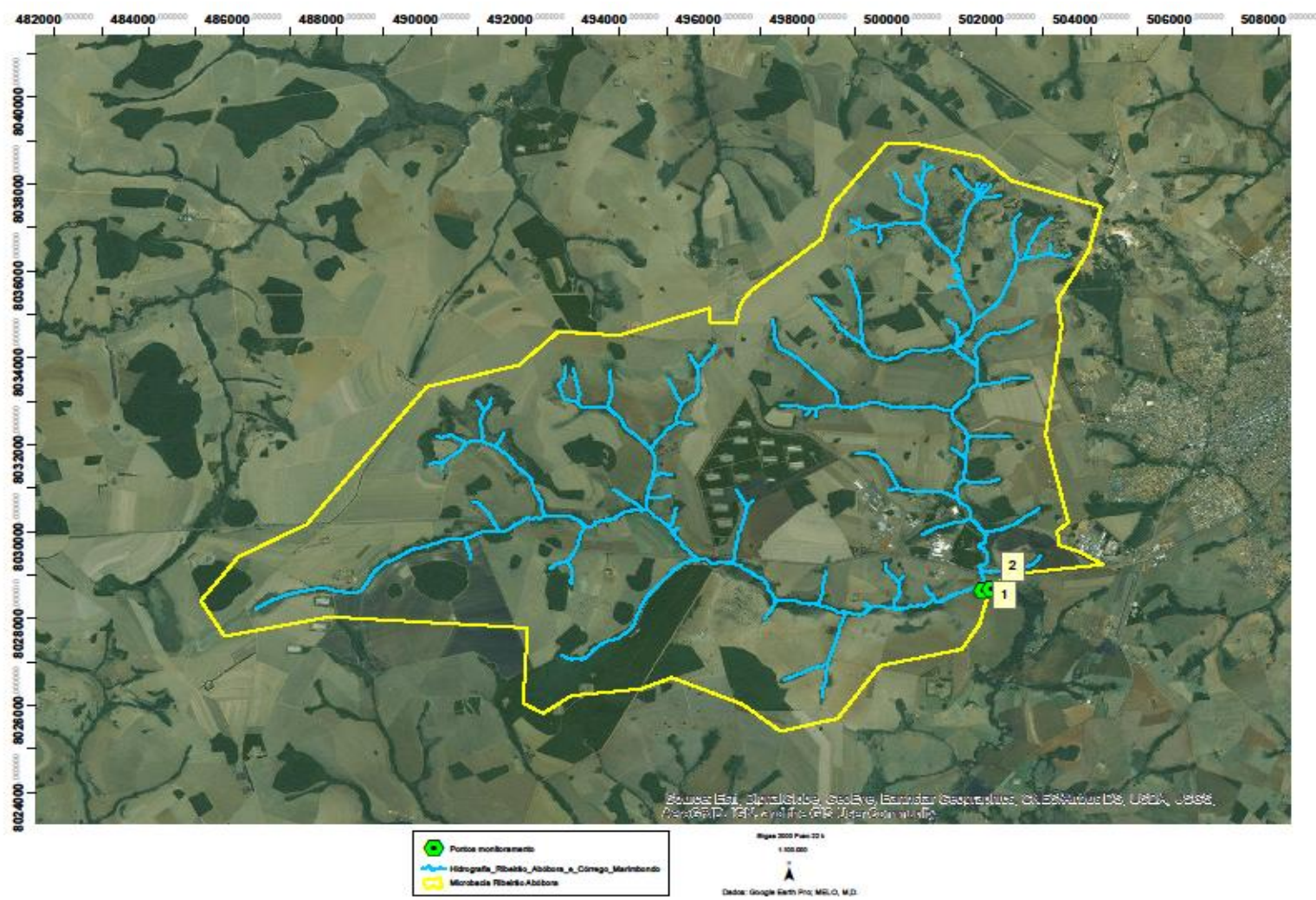

Fonte: Dados obtido através do Google Earth 2017.

As coletas de água foram realizadas em dois pontos do manancial, sendo a montante e a jusante do ponto de descarte de efluente existente nesse trecho do manancial. O ponto "1" localizado a montante do ponto de descarte de efluente com coordenadas $17^{\circ} 49^{\prime} 47^{\prime}$ 'S e $17^{\circ} 49^{\prime} 47^{\prime}$ 'S , e o ponto “2” situado a jusante do local de recebimento de esgoto a $17^{\circ} 49^{\prime} 45^{\prime}$ ' S e $50^{\circ} 58$ '55" O.

Foram realizadas cinco coletas de água, sendo 03 dessas nos meses de setembro, outubro e novembro de 2016 e 02 entre os meses de janeiro e fevereiro de 2017, totalizando 30 amostras. As preservações das amostras obedeceram aos procedimentos adotados na norma brasileira NBR9898 de junho de 1987, e os ensaios foram realizados de acordo com as técnicas recomendadas pelo Standard Methods for the Examination of the Water and Wastewater (2012).

Seguindo a metodologia abordada por Batista et al. (2017), a água monitorada deve receber tratamento conforme o enquadramento oferecido pelo CONAMA (357/2005), seu destino pode variar conforme a classe na qual se enquadrou, sendo:

- Classe 1 destina-se ao: abastecimento público para o consumo humano, após tratamento simplificado, as comunidades aquáticas, recreação e irrigação. 
- Classe 2 destina-se ao: abastecimento público para o consumo humano, após tratamento convencional, a proteção das comunidades aquáticas, irrigação, aquicultura e atividades pesqueiras.

- Classe 3 destina-se ao: abastecimento público para o consumo humano, após tratamento convencional ou avançado, irrigação, pesca, recreação (com restrições) e dessedentação de animais.

- Classe 4 destina-se à: navegação e harmonia paisagística.

Tabela 1. Classificação dos corpos hídricos com base na resolução Conama 357/05.

\begin{tabular}{ccccc}
\hline Parâmetro & Classe I & Classe II & Classe III & Classe IV \\
\hline $\mathrm{OD}$ & $\geq 6,0 \mathrm{mg} \cdot \mathrm{L}^{-1}$ & $\geq 5,0 \mathrm{mg} \cdot \mathrm{L}^{-1}$ & $\geq 4,0 \mathrm{mg} \cdot \mathrm{L}^{-1}$ & $\geq 3,0 \mathrm{mg} \cdot \mathrm{L}^{-1}$ \\
$\mathrm{NO}_{2}{ }^{-}$ & $\leq 1,0 \mathrm{mg} \cdot \mathrm{L}^{-1}$ & $\leq 1,0 \mathrm{mg} \cdot \mathrm{L}^{-1}$ & $\leq 1,0 \mathrm{mg} \cdot \mathrm{L}^{-1}$ & $\leq 0,07 \mathrm{mg} \cdot \mathrm{L}^{-1}$ \\
$\mathrm{NO}_{3}{ }^{-}$ & $\leq 10,0 \mathrm{mg} \cdot \mathrm{L}^{-1}$ & $\leq 10,0 \mathrm{mg} \cdot \mathrm{L}^{-1}$ & $\leq 10,0 \mathrm{mg} \cdot \mathrm{L}^{-1}$ & $\leq 0,40 \mathrm{mg} \cdot \mathrm{L}^{-1}$ \\
\hline
\end{tabular}

Fonte: Dados da própria pesquisa.

Os parâmetros de qualidade da água avaliados foram: nitrito $\left(\mathrm{NO}_{2}{ }^{-}\right)$, nitrato $\left(\mathrm{NO}_{3}{ }^{-}\right)$e oxigênio dissolvido (OD). Os parâmetros analisados assim como o método utilizado na análise dos mesmos em laboratório estão descritos na tabela 2.

Referente a estatística descritiva dos resultados, os cálculos do desvio padrão (DP) seguiram o descrito por Santos (2007) que estípula: - resultados iguais a "0,00" indicam que não há variabilidade exuberante dos valores em função da média; - resultados maiores que " 0,00 ”, indicam que há variabilidade dos valores em função da média. O cálculo do coeficiente de variação (CV) seguiu o demostrado por Pimentel Gomes (1985), correspondendo a: - CV até $10 \%$ - baixo (alta precisão para o objetivo); - CV de 0 a $20 \%$ - médio (média precisão); - CV 20 a 30\% - alto (baixa precisão); - CV > 30\% - muito alto (muito baixa precisão).

Tabela 2. Parâmetros físico-químicos avaliados e metodologia de análise.

\begin{tabular}{ccc}
\hline Parâmetros & Unidade de medida & Metodologia \\
\hline Oxigênio dissolvido & $\mathrm{mg} \cdot \mathrm{L}^{-1}$ & Oxímetro \\
Nitrito & $\mathrm{mg} \cdot \mathrm{L}^{-1}$ & Titulometria \\
Nitrato & $\mathrm{mg} \cdot \mathrm{L}^{-1}$ & Titulometria \\
\hline
\end{tabular}

Fonte: Dados da própria pesquisa.

\section{RESULTADOS E DISCUSSÕES}

A média geral dos valores de OD observados correspondeu a 6 mg.L $\mathrm{L}^{-1} \mathrm{e} 7 \mathrm{mg} \cdot \mathrm{L}^{1}$ para os anos de 2016 e 2017 respectivamente. Os meses de outubro/16 no ponto "jusante" e novembro/16 nos pontos "montante e jusante" apresentaram concentrações de OD abaixo do valor exigido $(\geq 5,0$ mg.L' 1) pela norma Conama 357/2005 para rios classe II, em que se enquadra o presente manancial estudado. Levando em consideração que existe descarte de efluente industrial próximo aos pontos monitorados, a demanda de OD é essencial para que a degradação da matéria orgânica ocorra de 
forma rápida e eficiente, levando em consideração também que algumas espécies de peixes não sobrevivem em ambientes aquáticos com teores de OD inferiores a 4,0 mg.L $\mathrm{L}^{-1}$ (MERCANTE et al., 2014).

Referente aos baixos valores de OD obtidos durantes os meses supracitados, segundo Souza et al. (2014), em que encontrou em dois pontos avaliados no Rio Almada (Sul da Bahia), valores de OD pertencentes a classe 3 da resolução citada. $\mathrm{O}$ autor explica que, os teores estão diretamente relacionados a contaminação antrópica, por parte de efluentes sanitários e domésticos, semelhante a presente pesquisa, em que os valores mais baixos de OD analisados, tem relação com os locais de atividades antrópicas ativas do tipo "industrial'.

A concentração média de $\mathrm{NO}_{2}^{-}$nas amostras de água do manancial analisado foram de 0,1 mg.L $\mathrm{L}^{-1}$ e $0,04 \mathrm{mg} . \mathrm{L}^{-1}$ para os anos de 2016 e 2017. Não houve ultrapassagem do limite legal $(\leq 1,0)$ preconizado pela norma Conama 357/05, sendo possível observar que o padrão de concentração obtido para esse parâmetro atende a qualidade mínima exigida para corpos hídricos classe I. Nitrato e nitrito são duas espécies vitais envolvidas no ciclo do nitrogênio, que é um dos ciclos biogeoquímicos mais importantes no ambiente marinho, portanto a determinação precisa de nitrato e nitrito é altamente importante para fins de monitoramento ambiental, oceanografia e química analítica (FANG et al., 2019). Concentrações elevadas de $\mathrm{NH}_{4}{ }^{+}, \mathrm{NO}_{2}{ }^{-}$e $\mathrm{NO}_{3}{ }^{-}$, derivadas de atividades humanas, podem, portanto, estimular ou melhorar o desenvolvimento, manutenção e proliferação de produtores primários (fitoplâncton, algas bentônicas, macrófitas), contribuindo para o fenômeno generalizado da eutrofização cultural (artificial) de ecossistemas aquáticos (CAMARGO; ALONSO; 2006) causa os altos teores de $\mathrm{NO}_{3}{ }^{-}$em água e ou alimentos.

Os resultados médios de $\mathrm{NO}_{3}{ }^{-}$para toda a campanha de monitoramento foi de $0,3 \mathrm{mg} . \mathrm{L}^{-1}$ para ambos os anos 2016 e 2017. Durante o monitoramento desse parâmetro foi observado uma “estabilização" das concentrações do poluente por todo o tempo de estudo. É possível que no trecho do manancial estudado, o estágio de redução da "amônia a nitrato, passando por nitrito" seja realizado de forma eficiente. Não houveram valores fora do padrão $\left(10,0 \mathrm{mg} \cdot \mathrm{L}^{-1}\right)$ legal estipulado pela norma Conama 357/05.

É importante ressaltar que esse agente orgânico participa do ciclo do nitrogênio e que na ocorrência de altos valores desse, existe a tendência da possibilidade de ocorrência problemas sérios como eutrofização de corpos hídricos. No caso do nitrato, a principal ação tóxica em animais aquáticos, principalmente em peixes e lagostins, parece ser a conversão de pigmentos portadores de oxigênio (hemoglobina, hemocianina) em formas que são incapazes de transportar oxigênio (methemoglobina, metemocianina) (CHENG; JUEN; CHENG; 2002). $\mathrm{O} \mathrm{NO}_{3}{ }^{-}$é fortemente monitorado também no Brasil, devido a comprovação de ocorrência da metemoglobinemia em 
crianças recém-nascidas, onde Machado et al. (1981) relatam em hospital do Ribeirão Preto, 26 casos acometidos com essa doença, que tem como causa os altos teores de $\mathrm{NO}_{3}{ }^{-}$em água e ou alimentos.

Figura 1. Monitoramento dos parâmetros $\mathrm{OD}, \mathrm{NO}_{3}{ }^{-}$e $\mathrm{NO}_{2}{ }^{-}$em 2016 e 2017.

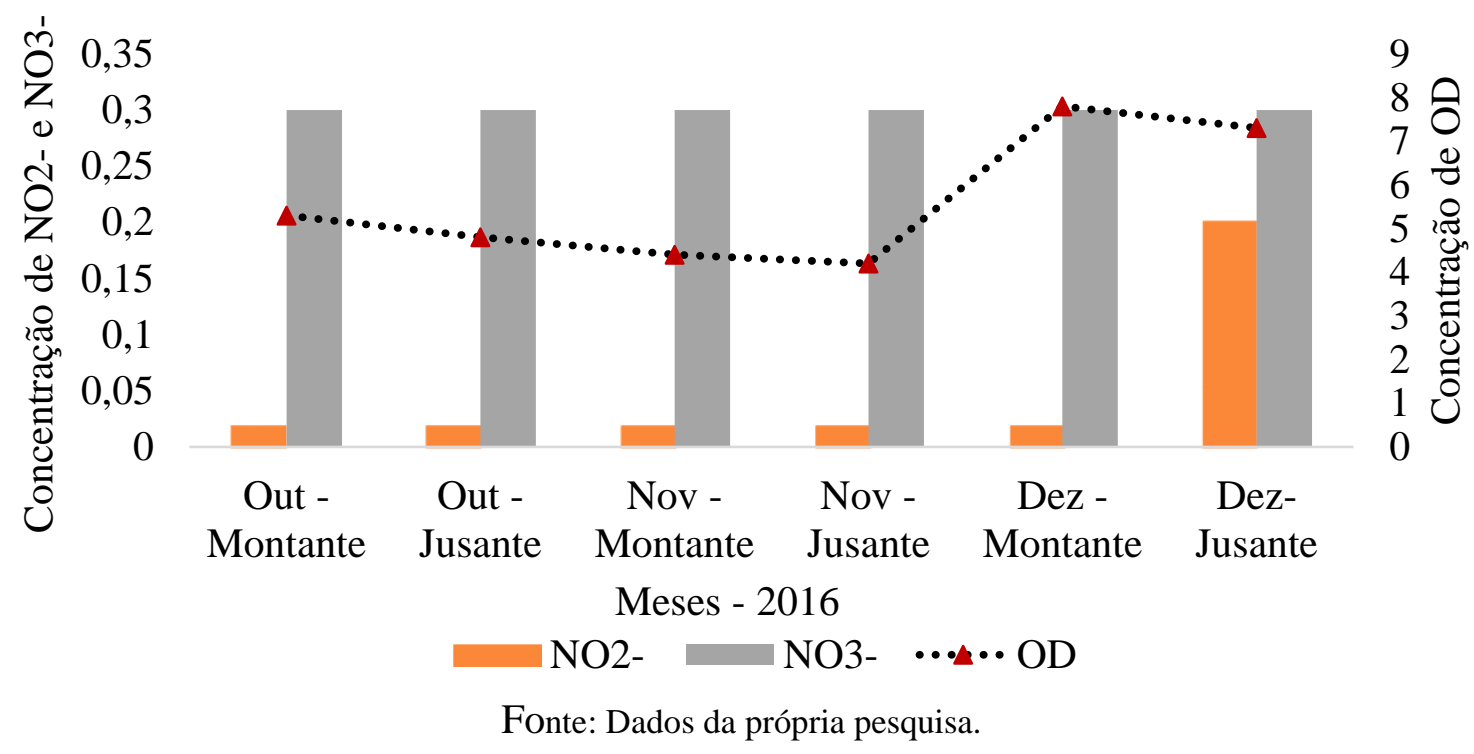

Figura 2. Monitoramento dos parâmetros $\mathrm{OD}, \mathrm{NO}_{3}{ }^{-}$e $\mathrm{NO}_{2}{ }^{-}$em 2016 e 2017.

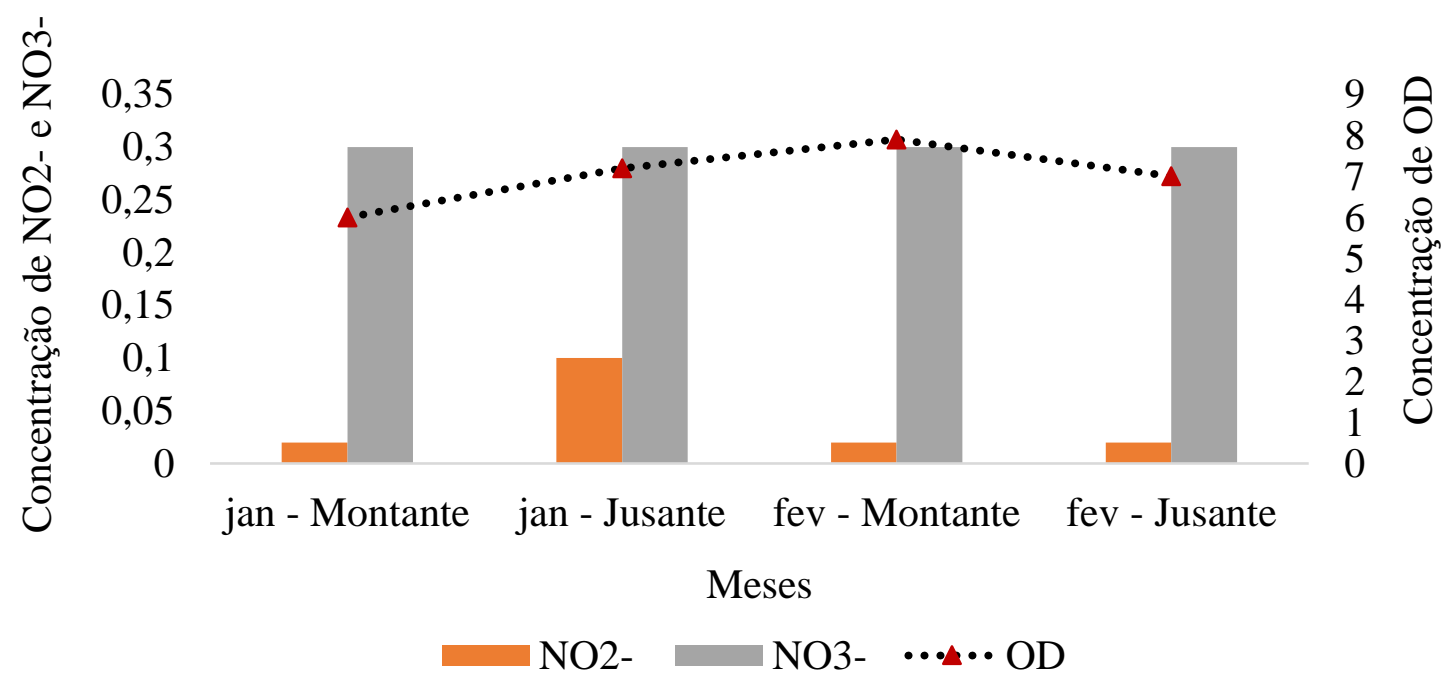

Fonte: Dados da própria pesquisa.

As análises estatísticas (tabela 3) dos valores obtidos para os parâmetros monitorados no ribeirão Abóbora, demonstram que somente para o OD no ano de 2016 houve desvio padrão (DP) superior a 1, sendo que em 2017 as médias dos resultados corroboraram para um DP igual a 0,78. Dessa forma, houveram variação dos dados em relação à média com base no desvio padrão. $\mathrm{O}$ coeficiente de variação $(\mathrm{CV})$ que dá uma noção a respeito da variação dos resultados em relação à média geral e o desvio padrão, apresentou comportamento inferior a 1,0 para toda a campanha de monitoramento, sendo possível afirmar que o monitoramento dos parâmetros de qualidade da água 
demonstra que muito baixa foi a variação dos resultados em relação à média geral dos resultados e ao desvio padrão desses.

Tabela 3. Estatística descritiva dos parâmetros monitorados.

\begin{tabular}{|c|c|c|c|c|c|}
\hline \multicolumn{6}{|c|}{ Estatística descritiva } \\
\hline Parâmetro & Mínimo & Máximo & Média & DP & $\mathrm{CV}$ \\
\hline \multicolumn{6}{|c|}{2016} \\
\hline OD & $4,20 \mathrm{mg} \cdot \mathrm{L}^{-1}$ & $7,80 \mathrm{mg} \cdot \mathrm{L}^{-1}$ & $5,60 \mathrm{mg} \cdot \mathrm{L}^{-1}$ & 1,54 & 0,27 \\
\hline $\mathrm{NO}_{2}^{-}$ & $0,02 \mathrm{mg} \cdot \mathrm{L}^{-1}$ & $0,20 \mathrm{mg} \cdot \mathrm{L}^{-1}$ & $0,10 \mathrm{mg} \cdot \mathrm{L}^{-1}$ & 0,07 & 0,73 \\
\hline $\mathrm{NO}_{3}{ }^{-}$ & $0,30 \mathrm{mg} \cdot \mathrm{L}^{-1}$ & $0,30 \mathrm{mg} \cdot \mathrm{L}^{-1}$ & $0,30 \mathrm{mg} \cdot \mathrm{L}^{-1}$ & 0,00 & 0,00 \\
\hline \multicolumn{6}{|c|}{2017} \\
\hline OD & $6,0 \mathrm{mg} \cdot \mathrm{L}^{-1}$ & 7,90 mg. $\mathrm{L}^{-1}$ & $7,00 \mathrm{mg} \cdot \mathrm{L}^{-1}$ & 0,78 & 0,11 \\
\hline $\mathrm{NO}_{2}^{-}$ & $0,02 \mathrm{mg} \cdot \mathrm{L}^{-1}$ & $0,10 \mathrm{mg} \cdot \mathrm{L}^{-1}$ & $0,04 \mathrm{mg} \cdot \mathrm{L}^{-1}$ & 0,04 & 1,00 \\
\hline $\mathrm{NO}_{3}^{-}$ & $0,30 \mathrm{mg} \cdot \mathrm{L}^{-1}$ & $0,30 \mathrm{mg} \cdot \mathrm{L}^{-1}$ & $0,30 \mathrm{mg} \cdot \mathrm{L}^{-1}$ & 0,00 & 0,00 \\
\hline
\end{tabular}

Fonte: Dados da própria pesquisa.

\section{CONCLUSÃO}

Os parâmetros $\mathrm{NO}_{2}{ }^{-}$e $\mathrm{NO}_{3}{ }^{-}$atenderam as exigências legais quanto as concentrações máximas permissíveis para mananciais classe II. Embora a média geral dos dados de OD monitorados durante toda a campanha, atenda a legislação ambiental, houveram 03 registros de concentrações inferiores ao padrão legal, resultado esse que chama a atenção a gestão do manancial nesse trecho em estudo, levando em conta a existência do descarte de efluente industrial que ocorre próximo aos pontos monitorados. Segundo o método abordado, quanto a variação dos dados observados o experimento pode ser classificado como de "boa precisão".

\section{REFERÊNCIAS BIBLIOGRÁFICAS}

AMERICAN PUBLIC HEALTH ASSOCIATION. Standard methods for the examination of water and wastewater. 22. ed. New York: United Book, 2012.

BATISTA, D.F.; CABRAL, J.B.P.; ROCHA, T., BARBOSA, G.R. Avaliação do oxigênio dissolvido nas águas do ribeirão paraíso em Jataí-GO e córrego tamanduá em Iporá-GO. Revista: Caminhos de Geografia, v.18, n.64, p.296-309, 2017.

CAMARGO, J.A.; ALONSO, A. Efeitos ecológicos e toxicológicos da poluição inorgânica do nitrogênio em ecossistemas aquáticos. Revista: Environment International. v.32, p.831 - 849, 2006. 
CHENG, S.Y.; JUEN, S.; CHENG, T.J.C.C. Acúmulo de nitrato nos tecidos de Penaeus monodon após exposição elevada a nitrato ambiente após diferentes períodos de tempo. Revista: Aquat Toxicol, v.56, p. $133-146,2002$.

FANG, T.; LI, P.; LIN, K.; CHEN, N.; JIANG, Y.; CHEN, J.; YUAN, D.; MA, J. Análise em andamento simultânea de nitrato e nitrito em águas estuarinas e costeiras usando um analisador de água ambiental automatizado e integrado à base de bomba de seringa. Revista: Analytica Chimica Acta, v.1076, p.100-109, 2019.

GARCIA, A.V.; OLIVEIRA, E.A.; SILVA, G.P.; COSTA, P.P.; OLIVEIRA, L. Disponibilidade hídrica e volume de água outorgado na micro-bacia do Ribeirão das Abóboras, município de Rio Verde, estado de Goiás. Revista: Caminhos de Geografia, v.8, n.22, p.97 - 106, 2007.

MAIA, K.P.; SILVA, G.A.D.; LIBÂNIO, M. Aplicação de análise multivariada no estudo da frequência de amostragem e do número de estações de monitoramento de qualidade da água. Revista: Eng.Sanit. Ambeint., v.24, n.05, 1013-1025, 2019.

MACHADO, N.C.; MACHADO, C.S.; MARTINEZ, F. E.; SOUZA, N.M. Surto de metemoglobinemia na enfermaria de pediatria do HCRP. Relato de 26 casos. / An outbreak of methemoglobinemia in the pediatric unit of the l"Hospital das Clinicas da Faculdade de Medicina de Ribeirao Pretol". Report of 26 cases. Revista: Medicina (Ribeiräo Preto);14(3/4):39-42, 1981.

MARANHO, A.L.; BOTELHO, G.R.; NOGUEIRA, A.L.; TORNISIELO, L.V. Avaliação da qualidade da água do Ribeirão Samambaia (São Pedro, São Paulo, Brasil) através de parâmetros físicos e químicos, índice de estado trófico e teste de toxicidade com Daphnia magna. Revista: Brasileira de Engenharia Sanitária e Ambiental. Piracicaba - SP, v.22, n.1, p.195-201,2017.

MERCANTE, C.T.J.; SANTOS, A.M.V.; MORAES, M.D.A.B.; PEREIRA, J.S.; LOMBARDI, J.V. Bullfrog (Lithobates catesbeianus) farming system: water quality and environmental changes. Revista: Acta Limnologica Brasiliensia. ISSN 2179-975X - 2014.

PIMENTEL, G.F. Curso de Estatística Experimental. São Paulo: Nobel, 467 p.; 1985.

SANTOS, C. Estatística Descritiva - Manual de Auto-aprendizagem, Lisboa: Edições Sílabo, 2007. SOUZA, J.R.; MORAES, M.E.B.; SONODA, S.L.; SANTOS, H.C.R.G. A Importância da Qualidade da Água e os seus Múltiplos Usos: Caso Rio Almada, Sul da Bahia, Brasil. REDE. Revista: Eletrônica do Prodema, Fortaleza, Brasil, ISSN: 1982-5528, v.8, n.1, p. 26-45, abr. 2014.

VANZELA, L.S.; HERNANDEZ, F.B.T.; FRANCO, R.A.M. Influência do uso e ocupação do solo nos recursos hídricos do córrego Três Barras, Marinópolis. Revista: Brasileira de Engenharia Agrícola e Ambiental. Campina Grande, v.14, n.1, p.55-64, 2010. 